\title{
Mandibular Advancement Devices (MAD) in the Therapy of Snoring and Obstructive Sleep Apnea (OSA)
}

\author{
Dr. med. Fahri Yildiz \\ Cologne Area, Germany
}

\section{Introduction}

Diagnosis and treatment of snoring and obstructive sleep apnea (OSA) is an interdisciplinary matter. In addition to Otorhinolaryngology it includes Internal Medicine, Pneumology, Cardiology, Dentistry, but also Pediatrics and Neurology. Continuous positive airway pressure (CPAP) is the primary noninvasive treatment of choice for OSA since its introduction in the early 1980s. Other alternatives include behavioral and surgical weight-loss therapies, pharyngeal and maxillomandibular surgeries, positional therapy, pharmacologic therapies, and mandibular advancement devices (MAD). The mandibular advancement devices (MAD) are now considered a reliable and non-invasive therapy option in the treatment of habitual snoring and obstructive sleep apnea (OSA) (Lit. 1, 2).

The American Academy of Sleep Medicine (AASM) has recognized and recommended MAD systems since 2006 . The position paper published in 2015 identifies MAD as a first line therapy for the patients with snoring and mild to moderate OSA (Apnea Hypopnea Index $[\mathrm{AHI}]<30 / \mathrm{h}$ ) and a Body Mass Index (BMI) of $<30 \mathrm{~kg} / \mathrm{m} 2$ ) in addition to Continuous Positive Pressure Breathing (CPAP) at night (Lit. 3, 4). It is known that CPAP therapy is not accepted by every patient. The CPAP-Compliance is less than $50 \%$ compared to up to $80 \%$ with MAD (Lit. 5). In the case of severe OSA (AHI > 30/h), CPAP treatment can be combined with MAD to reduce the respiratory pressure and thus increase acceptance (Lit. 3).

How do mandibular advancement devices work? MAD displace the lower jaw forward, making it more difficult to open the mouth and the tongue to fall back, thus increasing the volume of the upper airways. In addition, the hyoid is shifted upwards and forward and the velum is pulled forward by tightening the musculus palatoglossus. All this prevents collapse during sleep and reduces the obstruction.

The first studies on MAD in sleep apnea were conducted by Prof. Dr. Karlheinz Meier-Ewert, a German neurologist/psychiatrist and sleep physician, who first presented this form of therapy in 1984 (Lit. 5). For the nightly advancement of the lower jaw he used Monoblock devices, which he called Esmarch's device.

The MADs have undergone a positive development in recent years, namely from the one-piece chunky Esmarch device to elegant small two-piece models. With the two-piece MADs, the top and bottom trays are connected with different fasteners, such as frontal screws or hooks, side connectors or telescopes. This allows them to slide against each other and unlike the monobloc devices after adjustment the lower jaw can be moved forward until the desired effect is achieved. 


\section{Prefabricated thermoplastic MAD}

The so-called „Boil \& Bite“ MADs have been available for many years, which are made of thermoplastic material of meanwhile very good quality and are relatively inexpensive (Figures $\mathbf{1}-\mathbf{4}$ ). The advantage of this material is that the trays are heated in a water bath and then formed by biting the patient on the warm and soft material according to the individual teeth and jaw formation and can thus be immediately adapted chair-side to the patient without any major effort. After cooling the trays in cold water, the trays keep their new form. The patient can start to use it in the same night. The fitting can also be done in an ENT-Office also from an experienced nurse.

These thermoplastic MADs are available as Monoblock or two-piece devices, like the SomnoGuard AP2 (Fig. 1). The new SomnoGuard SPX, for example, is much smaller than the conventional thermoplastic devices (Fig. 4). It offers a higher using comfort due to considerably less thermoplastic material and delicate design. The new material has a so-called shape memory effect, which allows the device to be adjusted repeatedly, as it returns to its original shape in hot water and can be reshaped. It fits to all sizes of jaws and encloses the front teeth only slightly, so that little pressure is exerted on the teeth, which again considerably increases the using comfort.

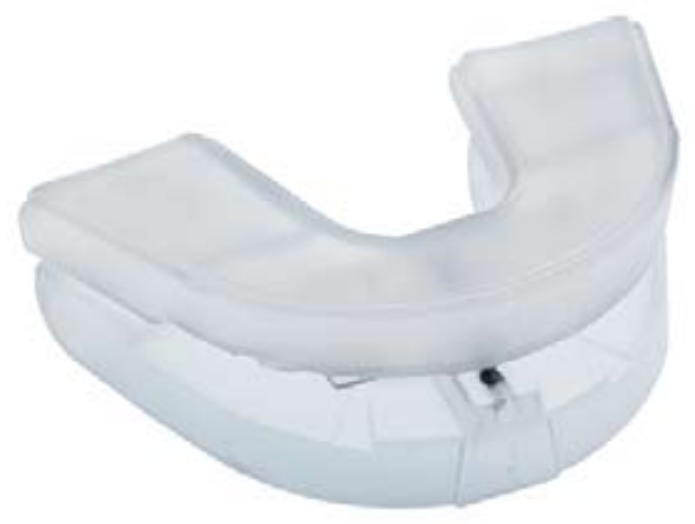

Fig. 1. SomnoGuard AP2

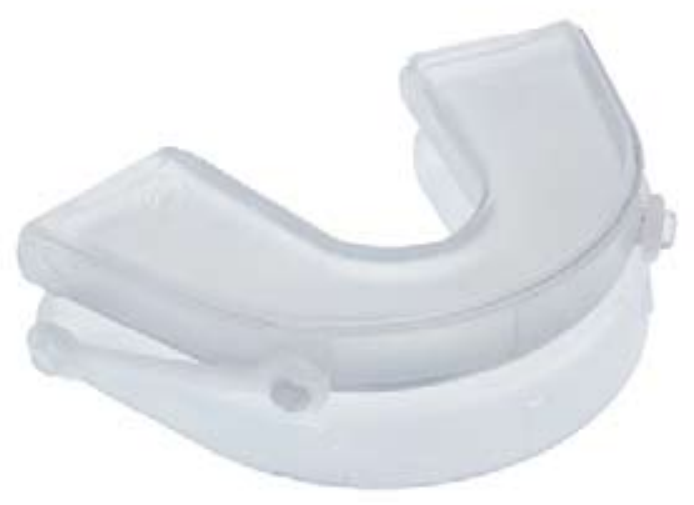

Fig. 2. SomnoGuard SP Soft

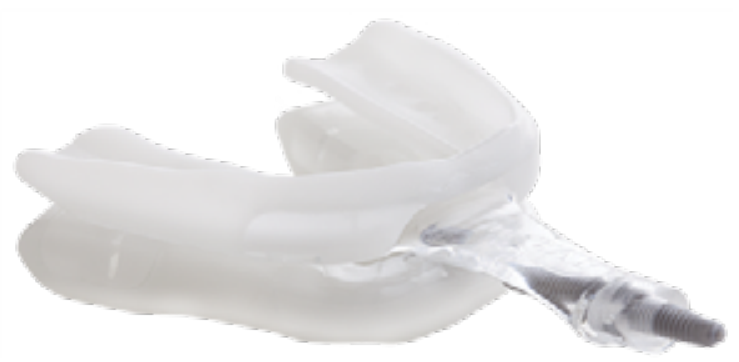

Fig. 3. My Tap

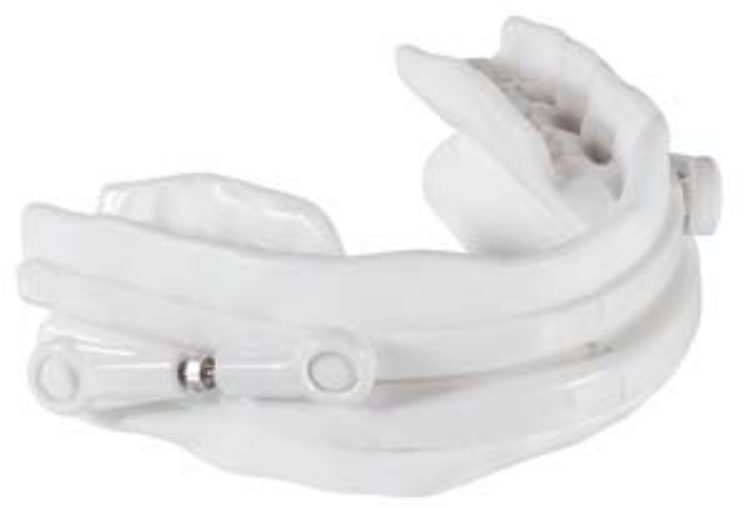

Fig. 4. SomnoGuard SPX

A further advantage is that the user can adjust the necessary mandibular advancement with millimeter precision even at the lateral connecting pieces (turnbuckle connecters or simple straps in different sizes. 
The thermoplastic MADs are also suitable as trial devices. Before investing in a more expensive custom MAD, it is recommended that you first select this less expensive option for testing. This will show whether the patient tolerates a MAD at all and whether the device is an effective therapy option for him. If its effectiveness can be proven over a certain period of time, the next step can be to use a MAD individually manufactured in the dental laboratory after the dentist has taken impressions. These custom-made devices are undoubtedly more comfortable and durable, but in contrast to thermoplastic devices they are significantly more expensive. This procedure offers the possibility to filter out „,non-responders“ of a MAD-Therapy and to avoid ineffective treatment. MAD must have the highest possible retention (adhesion) so that they can withstand the pull-off forces and remain in place on the rows of teeth at night. Only in this way can the lower jaw be reliably held in the protrusion position and an airway opening be guaranteed.

\section{Custom made MADs in the dental laboratory}

Depending on the findings, the dentist selects the most suitable device type for the patient (Figures 5 - 9). Individual impressions of the upper and lower jaws are required for the fabrication of the MAD in the dental laboratory. From these impressions, exact hard models are made so that the devices later fit exactly on the dental arch. In the past few years many of the dentists are using scan technic for the impressions which is more precise than a manual one. Then a special bite registration follows, in order to specify precisely to the dental technician in which mandibular advancement position the MAD should be made. Various factors such as the bite position (open bite, deep bite) and the inclination of the masticatory plane must be considered. Custom-made devices can be worn for up to 3 to 5 years, depending on the patient's care, oral hygiene and the presence of teeth grinding. Double layer materials are used for the newer ones. A softer and flexible inner lining provides a comfortable wearing comfort and the outer hard plastic layer provides more stability.

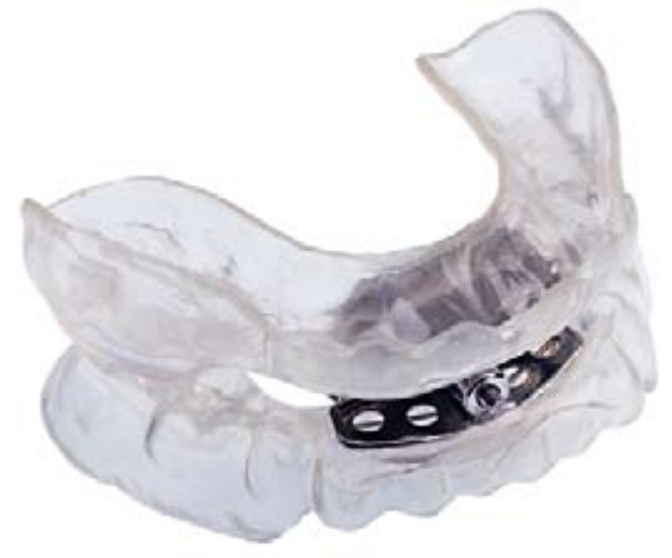

Fig. 5. SomnoGuard AP Pro

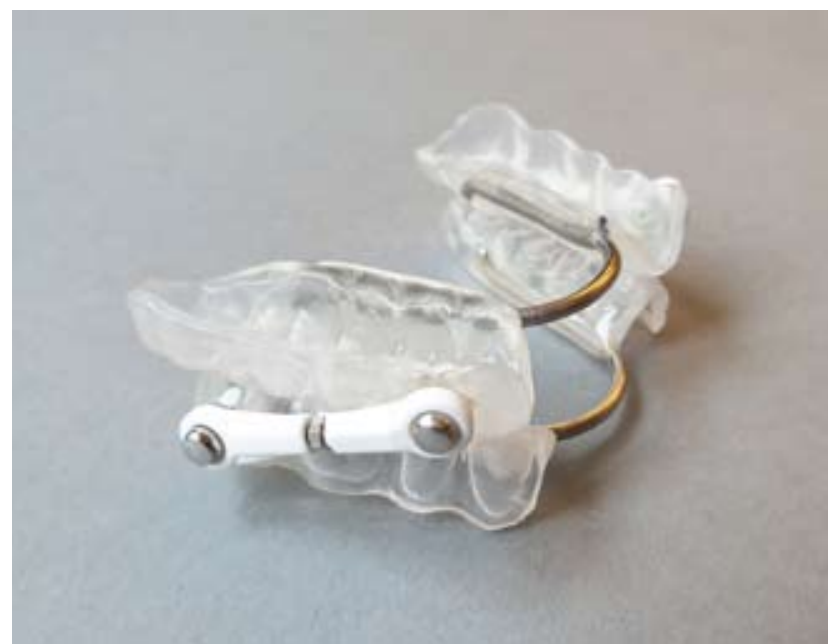

Fig. 6. SomnoGuard SP Pro

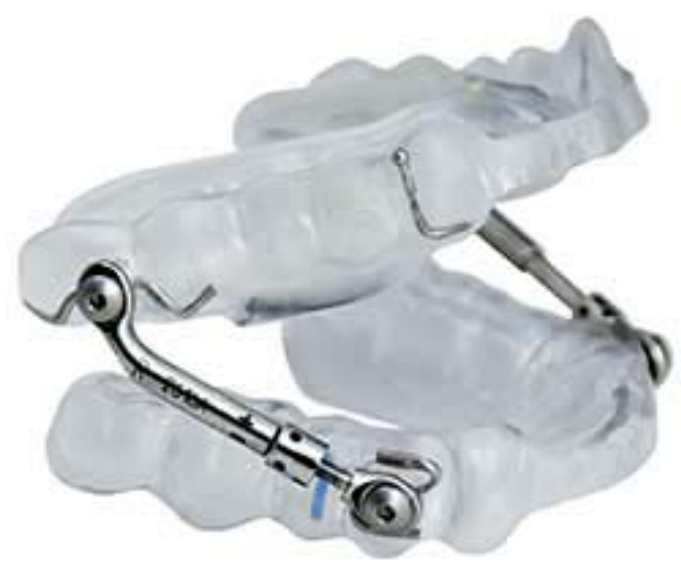

Fig. 7. Herbst Appliance 


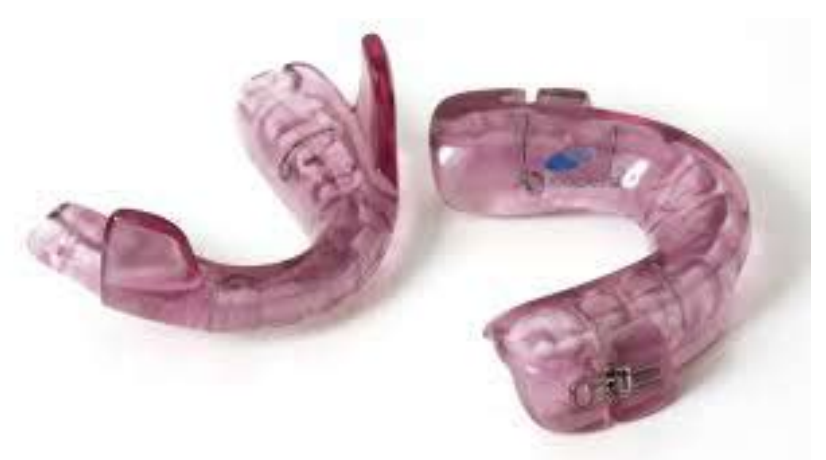

Fig. 8. SomnoDent

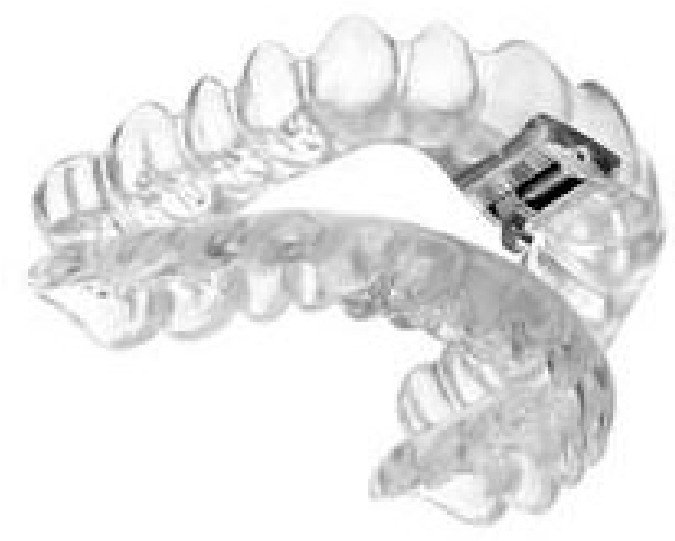

Fig. 9. TAP

Lately there are some newer nylon custom made devices in the market which are made with 3-D Printing technology after intraoral CAD/CAM Scan (Fig. 10).

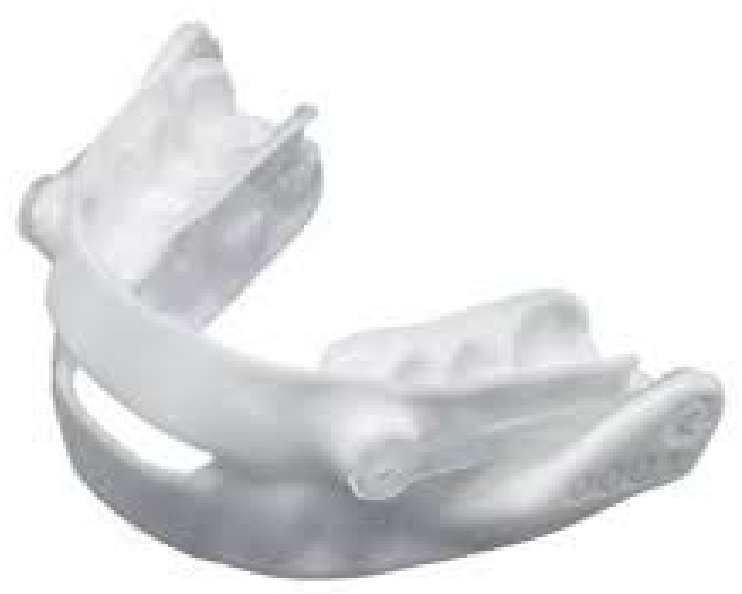

Fig. 10. Narval CC

\section{Examination before a fitting}

A thorough examination of the teeth, jaw and temporomandibular joints should be performed before a MAD fitting. This must be done to determine whether there are enough healthy teeth to anchor the devices. Existing periodontitis and caries must be detected and treated beforehand. If dentures are present, care must be taken to ensure that they are not damaged under the devices. The mobility of the temporomandibular joints at maximum advancement is measured and checked for possible pain. The findings of the buccal mucosa, tongue size in relation to the oral cavity, pharyngeal findings (soft palate, uvula, Mallampati degree) and the size of the tonsils should also be documented. A progeny or retrogene is to be recorded in the form of a photo documentation.

At the beginning of the treatment, the mandibular advancement should be about half of the maximum possible advancement. With increasing habituation, the advancement can be increased step by step up to two thirds of the maximum possible advancement, if necessary, to increase the effect. During the acclimatization period, which can last up to two weeks, hypersalivation, xerostomia, temporomandibular joint problems and toothache are possible. In very rare cases, permanent tooth misalignment may occur (Lit. 7).

\section{Controls after the fitting}

For OSA patients, outpatient monitoring (Polygraph) should be performed within three months of the start of MAD therapy in order to check the treatment outcome and whether the therapy goal has been achieved. If necessary, the setting of the MAD is changed and the lower jaw is moved further forward.

\section{Conclusion}

MAD is an evidence-based and internationally guideline compliant therapy for the interdisciplinary treatment of snoring, mild to moderate obstructive sleep apnea and patients with CPAP intolerance. It can be used either as first-choice therapy, as a treatment alternative in case of CPAP intolerance or together with CPAP as a combination therapy. For an effective MAD therapy in obstructive sleep apnea, it is recommended that less-cost prefabri- 
cated thermoplastic MAD are first used for testing to determine whether the patient tolerates a MAD and whether the device is an effective therapy option for him. Only then should a more elaborate and expensive MAD be used, which is individually manufactured according to impressions and adapted by dentists trained in sleep medicine.

\section{Literature}

1. Aarab G, Lobbezoo F, Hamburger HL, Naeije M. Effects of an oral appliance with different mandibular protrusion positions at a constant vertical dimension on obstructive sleep apnea. Clin Oral Investig 2010;14:339-345.

2. Almeida FR, Lowe AA. Principles of oral appliance therapy for the management of snoring and sleep disordered breathing. Oral Maxillofac Surg Clin North Am 2009;21: 413-420.

3. Randerath WJ et al. Konsensuspapier zur Diagnostik und Therapie schlafbezogener Atmungsstörungen bei Erwachsenen. Pneumologie 2014;68:106-123.

4. Kushida CA, Morgenthaler TI, Littner MR et al (2006) Practice parameters for the treatment of snoring and obstructive sleep apnea with oral appliances: an update for 2005. Sleep 29(2):240-243

5. Pantin CC1, Hillman DR, Tennant M., Dental side effects of an oral device to treat snoring and obstructive sleep apnea Sleep. 1999 Mar 15;22(2):237-40.

6. Meier-Ewert K, Schдfer H, КloЯ W (1984) Treatment of sleep apnea by a mandibular protracting device. 7th Europ Sleep Res Soc Congress, Munich (Abstract)

7. Menn SJ, Loube DI, et al. The mandibula repositional device: role in the treatment of obstructive sleep apnea. Sleep 1996; 19: 794-800 